\title{
Arbor
}

\section{Cuatro efemérides matemáticas a la sombra de los griegos: Thābit ibn Qurra, Gerolamo Cardano, Pierre de Fermat y las Disquisitiones de Gauss}

\author{
Antonio J. Durán Guardeño
}

Arbor CLXXI, 673 (Enero), 1-27 pp.

Usando como hilo conductor la recuperación y superación del legado matemático -y científico- griego, se analizarán en esta colaboración cuatro importantes efemérides matemáticas: la muerte de Thābit ibn Qurra (901), los nacimientos de Gerolamo Cardano (1501) y Pierre de Fermat (1601) y la publicación de las Disquisitiones Arithmeticae de Karl F. Gauss (1801).

Con esta perspectiva, consideraremos las contribuciones de Thābit al primer renacimiento de la ciencia y filosofía griegas que se produjo a partir del siglo VIII de manos de los árabes. Gerolamo Cardano aparecerá como actor de otro Renacimiento -este lo solemos escribir con mayúscula-: el italiano; que en este caso culminó con la recuperación para la Europa Occidental de la matemática, filosofía y ciencia griegas, además del arte. La figura de Pierre de Fermat se mostrará decisiva en la creación durante el siglo XVII de las dos herramientas matemáticas que suponen, tras dos mil años de historia, la superación conceptual y operativa de la matemática griega: la geometría analítica y el cálculo diferencial. Aunque en otra medida que Thābit, Cardano y Fermat, las Disquisitiones también pueden conectarse con el espiritu del mundo griego; fue, de hecho, el mismo Gauss quien lo invocó para darle realce y significado a uno de los principales resultados de su libro -el estudio de los polígonos regulares que admiten ser construidos con regla y compás-: «Es ciertamente atónito que nada nuevo se haya añadido a esta teoría desde los tiempos de Euclides hace ya 2.000 años». 


\section{Antonio J. Durán Guardeño}

\section{Introducción}

Se celebran en 2001 cuatro importantes efemérides de contenido matemático: la muerte de Thābit ibn Qurra (901), los nacimientos de Gerolamo Cardano (1501) y Pierre de Fermat (1601) y la publicación de las Disquisitiones Arithmeticae de Karl F. Gauss (1801). Aunque las efemérides hay que tomarlas como vienen se puede encontrar, afortunadamente, un fondo común tras estos cuatro acontecimientos aparentemente desconectados: todos tienen que ver, de una $\mathrm{u}$ otra forma, con la recuperación y superación del legado matemático griego. Este nexo de unión que afecta principalmente a las figuras de Thābit, Cardano y Fermat, y en menor medida a las Disquisitiones de Gauss, es bastante esclarecedor de lo que fueron las matemáticas hasta finales del siglo XVII $\mathrm{y}$, también, de lo que significan como ciencia, de su seña de identidad frente a las otras disciplinas científicas. Adoptaré, por esta razón, un enfoque comparativo más enriquecedor aunque respetando la singularidad de cada evento.

El punto de partida de esta historia será pues el ocaso de la ciencia griega. Lo podemos simbolizar en dos momentos históricos decididamente dramáticos -más desde luego el primero-. Por un lado, la muerte de Hipatia en 415 a manos de las turbas cristianas que acabó con siete siglos de ciencia en Alejandría y, por otro, el cierre decretado por Justiniano en 529 de todas las escuelas filosóficas griegas, incluida la Academia de Platón. Una parte de los filósofos y científicos afectados buscó refugio en la Persia de los sasánidas: la simiente que dejaron se mantuvo en estado latente hasta que empezó a florecer tras la rápida conquista musulmana del siglo VII. De todas las anexiones árabes me interesa aquí reseñar la de la ciudad de Harran, por su importancia en la historia de la ciencia y en una de nuestras efemérides matemáticas en particular. Harran está situada en la cabecera del río Eufrates -al sureste de la actual Turquía- y, según la Biblia, allí fue donde Abraham tuvo la inspiración de su fe monoteísta. A principios del siglo VIII d. C., poco después de ser conquistada por los árabes, Harran tras vencer la competencia de Antioquía se convirtió brevemente en la heredera de la tradición científica y cultural de Alejandría -digámoslo así-. Aproximadamente un siglo después, en 824-836 según otras fuentes- nació en Harran el primero de los personajes que aquí nos ocupa: Thābit ibn Qurra. Casi al mismo tiempo el califa abásida Al-Mamun fundó en Bagdad la Casa de la Sabiduría, el principal centro creador y difusor de la ciencia y la cultura árabes. En esos inicios del siglo IX se empezaron a traducir al árabe las primeras obras griegas: fue el inicio del primer renacimiento de la ciencia y filo- 
sofía griega. Como veremos más adelante, Thābit ibn Qurra contribuyó con varias traducciones a este renacer.

Desde este punto de vista, la conexión de Thābit con Cardano parece ahora clara: Gerolamo Cardano fue actor de otro Renacimiento -este lo solemos escribir con mayúscula-: el italiano; que en este caso culminó con la recuperación para la Europa Occidental de la matemática, filosofía y ciencia griegas, además del arte. Habrá ocasión más adelante de explorar estas interesantes similitudes. El prestigio matemático de Cardano tiene que ver justamente con el principal logro matemático del Renacimiento, ajeno por otra parte a la tradición griega: el álgebra, y en menor medida con la aritmética -esto es, el sistema decimal de numeración y los correspondientes algoritmos para realizar las operaciones básicas de sumar, multiplicar, dividir, extraer raíces cuadras, etc.; fue la gran aportación de la Edad Media a las matemáticas y, de nuevo, ajena al legado griego-.

Conviene recordar aquí, por matizar el párrafo anterior, que la recuperación del legado científico y filosófico griego en la Europa Occidental tardó varios siglos. El Renacimiento supuso una culminación del proceso, a la que no fue ajena la capacidad de difusión lograda tras la invención de la imprenta y la abundancia de material resultado de la huida cristiana tras la toma de Constantinopla por los turcos. Las traducciones de los griegos al latín comenzaron en el siglo XII principalmente en dos focos: Toledo y Sicilia, ambos en zonas de contacto con el mundo árabe, idioma a través del cual se recuperaron en primera instancia -y algunos en única- los clásicos -como característica propia del Renacimiento se acentuó el deseo de conocerlos de forma directa prescindiendo del filtro árabe-. En lo que atañe a las matemáticas se puede afirmar que a finales del siglo XVI, el siglo en que vivió Cardano, la recuperación del legado griego distaba todavía de ser satisfactoria; aunque la geometría elemental de Los Elementos era dominada a la perfección por los matemáticos del Renacimiento, no ocurría lo mismo con la asimilación de la matemática superior y profunda de Arquímedes. Aunque la edición príncipe de las obras de Arquímedes apareció en 1544, no fue hasta casi un siglo después cuando los matemáticos europeos fueron capaces de asimilarlo e igualar sus logros -cosa que, por ejemplo, sí había conseguido Thābit isiete siglos antes!-. La situación era distinta en otros aspectos de la ciencia: por ejemplo, en astronomía la total asimilación del legado griego se produjo durante el siglo XV; en ese siglo, tanto Peurbach como Regiomontano, por citar los autores más significativos, ya dominaban perfectamente la sofisticación técnica de la astronomía ptolemaica. Y, un año antes de la publicación de la edición príncipe de las obras de Arquí- 
medes, apareció el De revolutionibus de Copérnico que, aunque todavía contaminado -Kepler dixit-por el espíritu ptolemaico, generó las consecuencias revolucionarias que todos conocemos bien. Sin querer extender la comparación a otros ámbitos, pero por lo que de significativo tiene y la relación que guarda con Cardano -no olvidemos que también fue médico-, no dejaré de recordar que ese mismo año -1543- apareció también el De humani corporis fabrica de Vesalio.

Justo en ese momento histórico en que los matemáticos occidentales empiezan a superar a los griegos en aquello en que estos seguían siendo los maestros -primera mitad del siglo XVII-, encontramos al tercero de nuestros personajes: Pierre de Fermat. Fermat contribuyó de manera decisiva a la creación durante el siglo XVII de las dos herramientas matemáticas que suponen, tras dos mil años de historia, la superación conceptual y operativa de la matemática griega: la geometría analítica y el cálculo diferencial. Ambos logros basados en aspectos matemáticos -habría que decir también metafísicos e, incluso, ontológicos- que los griegos dejaron casi vírgenes: la generalidad del álgebra -y en menor medida la aritmética- en el caso de la geometría analítica, y la potencia de los procesos infinitos junto con la consideración del infinito en acto -prohibido por Aristóteles- en el caso del cálculo.

Pero ¿y el último de los acontecimientos?, ¿podemos encadenar también esta joya -las Disquisitiones de Gauss- con los eslabones de oro de la matemática griega? Desde luego no en la misma medida en que Thābit, Cardano y Fermat lo han sido, pero algo puede hacerse. De hecho, es el mismo Gauss quien al final de las Disquisitiones invoca el espíritu del mundo griego para darle realce y significado a uno de los principales resultados de su libro: el estudio de los polígonos regulares que admiten ser construidos con regla y compás. Así, después de mostrar como se puede hacer la construcción de un polígono regular de 17 lados escribe ([Gauss, 1981: 462]):

«Es ciertamente atónito que, aunque la divisibilidad geométrica de una circunferencia en 3 y 5 partes fuera ya conocida en el tiempo de Euclides, nada fuera añadido a este descubrimiento en 2.000 años. $Y$ que todos los geómetras hayan afirmado que, excepto para este número de partes y las que se derivan directamente de ellas, no hay otras que puedan ser geométricamente construidas».

\section{Thăbit ibn Qurra}

Como ya hemos comentado Thābit ibn Qurra nació en Harran en 824 -836 según otras fuentes-. No son muchos sus datos biográficos conser- 
vados. Sabemos que perteneció a la secta de los sabeos adoradores de astros de Harran, que existieron hasta el siglo XI -no confundir con los mandeos, que tomaron el mismo nombre, pero son un secta gnóstica actualmente existente-. Precisamente su religión hizo que produjeran algunos buenos astrónomos, entre ellos Thābit -también Al-Battani (850929), o Albategnius como fue conocido durante la Edad Media-. En la época de Thābit todavía se conservaba en Harran un cierto dominio del griego que había sido impuesto como lengua oficial tras la conquista de la zona por Alejandro Magno -los habitantes de Harran también usaron entonces nombres griegos-; la lengua madre de la zona era el siríaco -dialecto originado tras la diversificación que sufrió el arameo como consecuencia de la presión del griego-. Durante el siglo VI hubo cierta tradición de traductores en Harran: se hicieron entonces traducciones del griego al siríaco de obras de Galeno y Aristóteles, entre otros autores. Después de la conquista árabe los habitantes de Harran hablaron también árabe y usaron nombres árabes. Toda esta tradición permitió a Thābit dominar las tres lenguas, lo que pudo ser decisivo para su incorporación a la Casa de la Sabiduría en Bagdad, donde estaban muy interesados en verter al árabe todo el acervo de ciencia griega del que empezaban a estar bien surtidos. Thābit que gozaba de cierto desahogo económico -en su juventud se dedicó al negocio del cambio de moneda lo que le permitió hacer cierta fortuna- se convirtió en Bagdad en un gran matemático y astrónomo, además de filósofo y médico. No mucho más se sabe de su vida: parece ser que tuvo algunos problemas con su secta por lo que se exilió definitivamente en Bagdad; allí, durante los últimos años de su vida, entró a formar parte del séquito del califa abásida al-Mutadid.

Pasaré ahora a comentar la obra científica de Thābit, aunque sin ánimo de ser exhaustivo -en cualquier caso, para los trabajos matemáticos véase su biografía en el Dictionary of Scientific Biography, y para los astronómicos el libro de Régis Morelon [Thābit, 1987] o, en su defecto, su artículo [Morelon, 1994]-.

Empezaré con las traducciones de Thābit del griego al árabe: darán una idea cabal de hasta que punto esta época, y las contribuciones de Thābit en particular, supusieron un renacimiento de la ciencia griega. En primer lugar hay que señalar la revisión y anotación de Thābit de las traducciones que de Los Elementos de Euclides y del Almagesto de Ptolomeo hizo Ishaq ibn Hunayn -se conservan cartas de Thābit al hijo de Ishaq-. Esa traducción árabe de Los Elementos, que sirvió posteriormente para la traducción al latín que hizo Gerardo de Cremona (1114-1187) en Toledo, se conserva todavía en varios manuscritos custodiados en la Biblio- 


\section{Antonio J. Durán Guardeño}

teca Bodleiana de Oxford. También la versión árabe del Almagesto, que se conserva en parte, fue probablemente usada por Gerardo de Cremona para hacer la primera traducción latina -el objeto de deseo que llevó a Cremona a Toledo-; casi cuatro siglos después (1515) esa traducción latina se publicó en Venecia: fue la primera impresión del Almagesto, uno de cuyos ejemplares perteneció precisamente a Copérnico. Thābit también tradujo obras de Arquímedes -Sobre la esfera y el cilindro- o atribuibles a Arquímedes; entre estas últimas el Libro de los lemas del que sólo nos han llegado versiones en árabe, una la de Thābit, que fue impresa varias veces en el siglo XVII. Algo parecido ocurrió con Las cónicas de Apolonio: de los 8 libros que formaban esta obra, sólo los cuatro primeros se conservan en griego, el octavo se considera perdido, y del quinto, sexto y séptimo sólo se conservan copias árabes, una la de Thābit. Finalmente también tradujo los Elementos de Geometría del astrónomo Menelao, hoy desaparecidos tanto en el original griego como en sus versiones árabes, y la Aritmética del neopitagórico Nicómaco de Gerasa.

Pero Thābit fue también autor de numerosos resultados en varias áreas distintas de las matemáticas. Como el lector podrá comprobar su obra supone una continuación de la matemática griega en el nivel máximo que esta alcanzó. Empezaré con un interesante resultado sobre teoría de números -la rama de las matemáticas que después refundaría Fermat, y a la que Gauss con las Disquisitiones elevaría a la categoría de arte-: se trata de un criterio para la determinación de números amigos ${ }^{1}$ que generaliza el criterio de Euclides para números perfectos ${ }^{2}$. Aparece en su tratado Sobre la derivación fácil de números amigos y es el siguiente: $\mathrm{Si}$ $\mathrm{p}=3 \times 2^{\mathrm{n}-1}-1, \mathrm{q}=3 \times 2^{\mathrm{n}}-1$ y $\mathrm{r}=9 \times 2^{2 \mathrm{n}-1}-1$ son números primos, con $\mathrm{n}>1$, entonces el par $2^{\mathrm{n}} \times \mathrm{p} \times q$ y $2^{\mathrm{n}} \times \mathrm{r}$ son números amigos. El caso $\mathrm{n}=2$ da el par 220 , 284 mencionado por Jamblico (s. IV d.C.) en sus comentarios a la Aritmética de Nicómaco. Para $\mathrm{n}=3,5,6,8$ y 9 no se verifican las hipótesis porque p, q y r no son todos primos. El caso $\mathrm{n}=4$ da el par 17296 y 18416 ; aunque las primeras referencias a este par aparecen en fuentes árabes del siglo XIV -siendo Fermat, precisamente, el primero en mencionarlo en fuentes occidentales-, Hogendijk lo ha adjudicado recientemente (1985) a Thābit -aunque el par no aparece específicamente en ningún tratado de Thābit_ ${ }^{3}$.

A Thābit también se deben algunos resultados en geometría elemental y aritmética geométrica: en particular varias pruebas generales del teorema de Pitágoras -en la línea de la que Platón atribuye a Sócrates en el Menón- y también una extensión que aparentemente no ha sido redescubierta hasta 1790: aparece en la edición de Montucla (1790) del libro de problemas recreativos: Récreations mathématiques et physiques de 
Jacques Ozaman -Montucla lo atribuye erróneamente al hermano menor de Alexis Clairaut; véanse los artículos de Carl B. Boyer [Boyer, 1964] y A. Sayili [Sayili, 1960] para la historia completa-; y lo más importante, varios intentos de demostrar el quinto postulado de Euclides -en ellos critica a Euclides por renunciar a la utilización del movimiento en geometría; por cierto, parece ser que Thābit fue el primer autor que habló de velocidad de movimiento en un punto (velocidad instantánea): concretamente en un tratado de astronomía en relación con la velocidad del sol en su apogeo y perigeo [Morelón, 1994: 113]-. También resolvió el problema de la trisección del ángulo usando, como Arquímedes, puntos de intersección de hipérbolas y circunferencias.

Pero Thābit también demostró un dominio de las técnicas más difíciles y duras de Arquímedes -como se dijo en la Introducción, nadie en Europa Occidental llegó a tener un dominio parecido hasta bien entrado el siglo XVII-: en concreto calculó el área de la parábola usando el método de exhaucción con un procedimiento equivalente al cálculo de $\int \sqrt{ } \mathrm{x} d \mathrm{x}$-en vez de $\int \mathrm{x}^{2} \mathrm{dx}$ como hizo Arquímedes-; también calculó áreas y volúmenes de cuerpos parabólicos, secciones cilíndricas, etc.

Finalmente, y como un subproducto de su actividad astronómica, Thābit produjo algunos resultados en trigonometría esférica y plana -a parte de introducir el uso del seno, invento hindú, en la trigonometría griega: el seno es la semicuerda del ángulo doble, mientras que los griegos habían usado la cuerda del ángulo-.

Muy interesantes son también sus reflexiones sobre el infinito -que cabe encuadrar entre sus aportaciones filosóficas-: aparecen en una discusión con su alumno cristiano Abu Musa ibn Usayd en el contexto de la controversia teológica relativa a la enumeración de las almas y al conocimiento por Dios de la infinidad de seres singulares -estos argumentos teológicos sobre el infinito reaparecen después en la escolástica medieval y en todos los grandes filósofos del siglo XVII; como veremos cuando tratemos a Fermat esas elucubraciones fueron esenciales para superar el horror infiniti que pareció aquejar a los griegos-. Thābit admitió contraviniendo a Aristóteles que el infinito en acto es admisible, la comparación entre infinitos es también posible -emparejando sus elementos-, y por tanto es posible establecer jerarquías de tamaño entre ellos. Aunque esto nos suena casi a Cantor, no conviene emocionarse más de la cuenta, aunque Thābit parece intuir las ideas que llevarían un milenio después a los matemáticos a domar el infinito en acto, el ejemplo que maneja es contradictorio y confuso: asegura que el infinito de los números naturales sería el doble que el infinito de los números pares -hay tantos números pares como impares, y juntos son todos los números-; sin embargo, el in- 


\section{Antonio J. Durán Guardeño}

finito de todos los números y el de los números pares es manifiestamente el mismo pues se puede emparejar cada número con un número par sin que sobren números ni falten números pares ${ }^{4}$.

Thābit fue también un gran astrónomo: aquí volvió a demostrar un conocimiento y dominio profundo, en este caso, de la astronomía griega, en especial de Ptolomeo. Su contribución astronómica más influyente fue en relación con la precesión de los equinoccios, esto es, el lento movimiento del polo celeste a través de las estrellas -completando una circunferencia al cabo de 26.000 años-. Este fenómeno fue importante porque afectaba a la duración del año trópico, esto es, el año de las estaciones y por tanto el que fija la duración del año a efectos de la elaboración de un calendario: como es bien sabido la medición exacta del año trópico para la reforma del calendario fue uno de los motivos que hicieron plantearse a Copérnico en el siglo XVI la reforma de la astronomía. Sin embargo, la aportación de Thābit fue errónea: propagó la falsa teoría que establecía que la precesión variaba en cuanto a su velocidad y dirección, esto es, la precesión era una especie de oscilación o trepidación respecto a una posición media. Esto se dio en llamar la teoría de la trepidación y parece ser que fueron Teón de Alejandría y Proclo sus primeros defensores -la precesión de los equinoccios, como explicaría después Newton es, en realidad, la consecuencia de la atracción gravitacional de la Luna sobre la protuberancia ecuatorial de la Tierra-. Thābit cotejó datos de observación de los griegos con los recientes obtenidos en los observatorios de $\mathrm{Da}$ masco y Bagdad -algunos de ellos erróneos-, y propuso un complicado sistema de nuevas esferas añadidas a la de las estrellas fijas para dar cuenta de la supuesta trepidación; la teoría aparece después recogida por Azarquiel (s. XI) en sus Tablas Toledanas -que derivaron, como es bien conocido, en las Alfonsinas- e incluso también en el libro tercero del $D e$ revolutionibus de Copérnico. Para descargo de Thābit es conveniente añadir que manifestó siempre cierta reserva sobre la teoría de la trepidación pensando que serían necesarias más observaciones para poder dilucidar su validez.

Otra aportación importante de Thābit fue en relación con el conflicto manifiesto entre la astronomía ptolemaica y la cosmología aristotélica -otro de los motivos que llevaron a Copérnico a plantearse la reforma de la astronomía-. En este sentido, Thābit se inclinó por acomodar la cosmología a la astronomía, frente a los astrónomos árabes y judíos que florecieron en Al-Andalus en el siglo XII -Avempace, Averroes, Alpetragio, Maimónides- que optaron por adecuar la astronomía a la cosmología.

Pero, además de matemático y astrónomo, Thābit fue también médico - uno de los mejores médicos del cercano Oriente medieval; escribió va- 
rios tratados sobre Galeno y medicina general-y naturalista -escribió también sobre anatomía de los pájaros, veterinaria y botánica-. Como se puede observar la figura de Thābit se adapta perfectamente a la del hombre del Renacimiento, como Cardano del que me paso a ocupar ahora -dejo para después la comparación de ambos-.

\section{Gerolamo Cardano}

Todo intento de reflejar la personalidad abrumadora de este genio del Renacimiento en unos breves párrafos es tarea imposible; innecesaria además disponiendo como disponemos de su colorista e inclasificable autobiografía: De propia vita -hay edición castellana en Alianza, de donde se han tomado las citas que siguen-. De manera que me contentaré con apuntar algunos hechos significativos de su vida procurando, en la manera de lo posible, tomarlos directamente de De propia vita. Empezaré por su nacimiento:

"Después de probar en vano diversos abortivos -según oí contar- nací el año 1501, el día 24 de septiembre, cuando todavía no había transcurrido integra la primera hora de la noche, sólo poco más de su mitad, aunque sin llegar todavía a las dos terceras partes» ([Cardano, 1991: 83]).

Cardano fue hijo ilegítimo - de ahí las referencias explícitas a su nacimiento no deseado- de un abogado amigo de Leonardo da Vinci y una viuda «irascible, inteligente, memoriosa, de baja estatura, gorda, devota» ([Cardano, 1991: 88]) -las referencias tan precisas al momento de su nacimiento las usa después para elaborar su horóscopo, del que más adelante se ofrecerá algún detalle-. Estudió primero en Pavía y después en Padua entre 1520 y 1526, año en que obtuvo el título de doctor en Medicina. Para el resto de apuntes biográficos me apoyaré en el siguiente catálogo de desgracias que describe en el capítulo Sobre mi mismo ([Cardano, 1991: 278]):

«Desgracias son la muerte -y muerte violenta-de uno de mis hijos, la vida disipada del otro, la esterilidad de mi hija, mi impotencia sexual, la constante pobreza, pleitos, acusaciones, percances, enfermedades, peligros, cárcel, la injusticia de verme postergado tantas veces y frente a tantos hombres inferiores a mí. Pero [...] no puedo con razón quejarme de mi suerte».

Añadamos ahora algunos detalles a ese esquema. «La injusticia de verme postergado tantas veces y frente a tantos hombres inferiores a mí» 
hace referencia a las varias veces -1529 y 1537 - que, por ser hijo ilegítimo, le fue impedido ejercer la medicina por el colegio de médicos de Milán hasta que fue finalmente admitido -1539-. En ese periodo ejerció, entre otras cosas, de profesor de matemáticas en una escuela de Milán. «Impotencia sexual» hace referencia a la que sufrió durante una etapa de su vida; sus causas las explica así en el horóscopo que sigue a la descripción de su nacimiento: dado que ese día «Júpiter estaba en ascendente junto con Venus, que dominaba todo mi horóscopo, no pasé sin cierto daño en mis genitales, de modo que desde los veintiún años a los treinta y uno no pude realizar el coito» ([Cardano, 1991: 85]); el mismo año de su curación se casó. «Cárcel»: Cardano fue encarcelado por la Inquisición en 1570 acusado de haber realizado el horóscopo de Jesucristo; después de abjurar fue liberado pero perdió la cátedra de medicina que en esos momentos ocupaba en la Universidad de Bolonia -el escándalo no impidió, sin embargo, que al año siguiente entrara al servicio del papa como astrólogo, entre otras cosas-. "Desgracias son la muerte -y muerte violenta- de uno de mis hijos», se refiere aquí Cardano a uno de los episodios más amargos de su vida: la condena y posterior ejecución de su hijo mayor acusado de haber envenenado a su esposa; «la vida disipada del otro, la esterilidad de mi hija»: pues eso, ninguno de sus tres hijos hizo la felicidad de Cardano, aunque en parte él se consideraba responsable: «Mi mayor equivocación estuvo en la educación de mis hijos, porque la educación puede mucho» ([Cardano, 1991: 308]). A pesar del catálogo de infortunios Cardano no fue del todo infeliz: «Aunque la palabra felicidad anda reñida con nuestra condición de hombres, sin embargo, por estar más cerca de la verdad decir que podemos, al menos en parte, ser dichosos, diré que también a mi modo lo he sido» ([Cardano, 1991: 187]).

Cardano siempre se enorgulleció de sus habilidades médicas -sobre todo de la curación del arzobispo de Edimburgo- y astrológicas que, además, le hicieron célebre en su época:

"Así pues, tres fueron las veces que en cincuenta y tres años de ejercicio de la medicina me he equivocado, mientras que Galeno no reconoció sus errores porque había cometido tantos, que el reconocerlos no le podía servir de excusa» ([Cardano, 1991: 203]).

Las siguientes citas son ilustrativas del carácter ambicioso, polémico, irónico y, también, juicioso de Cardano:

"La idea y el deseo de perpetuar mi nombre se apoderaron tan pronto de mí como tarde estuve en condiciones de aspirar a tal cosa. Y es que yo veía claramente que hay dos clases de vida, una elemental que compartimos con 


\section{Cuatro efemérides matemáticas a la sombra de los griegos...}

los animales y plantas y otra que es exclusiva del hombre, que lo que busca con sus acciones es gloria» ([Cardano, 1991: 112]).

"Admito como el mayor y más raro de mis extravios una irreprimible tendencia a no decir nada con más agrado que aquello que molesta a mis interlocutores. Persevero a sabiendas y gustoso en este defecto mío. No se me escapa cuántos enemigos me he granjeado» ([Cardano, 1991: 129]).

"De vez en cuando también me he visto afectado de enamoramientos heroicos hasta abrigar pensamientos suicidas. Pero tales cosas supongo que les ocurren a todos, aunque no las cuenten en los libros» ([Cardano, 1991: 104]).

"La amistad, incluso la falsa, no la rasgaré, sino que la descoseré» ([Cardano, 1991: 158]).

Cardano fue también singular como matemático: los dos resultados que le dieron la fama, la resolución de las ecuaciones de tercer y cuarto grado, no fueron ninguno de ellos obra suya. De los más de 200 libros que escribió tres fueron de matemáticas: Practica arithmetica (1539), Ars Magna (1545) y Liber de ludo aleae. El primero es una obra menor dedicado a la aritmética, aunque no hay que olvidar que la aritmética fue la gran aportación matemática de la Edad Media que el Renacimiento se encargó de difundir. El Liber de ludo aleae apenas tuvo transcendencia, a pesar de ser el primer libro sobre probabilidades, pues aunque escrito en 1564 no fue publicado hasta 1663, después de las cartas cursadas entre Fermat y Pascal; de cualquier manera refleja la pasión de Cardano por el juego -y sus trampas-, aunque: «no fue afición al juego la mía ni ansias de dinero, sino amargura y escapatoria».

Fue el Ars Magna, la gran síntesis del álgebra renacentista, el libro que hizo entrar a Cardano en la historia de las matemáticas. Como ya se ha apuntado, la gran aportación del libro, la resolución de las ecuaciones de tercer y cuarto grado, no fueron ninguna originales de Cardano. La historia de la disputa de Cardano -y Ferrari- con Tartaglia es una buena muestra del colorido de la época, pero como es bien conocida prescindiré de contarla aquí. En cualquier caso reproduciré el resumen que hace Cardano en el Ars Magna sobre quién descubrió la regla para resolver la cúbica:

"Scipione del Ferro, de Bolonia, hace más de 30 años inventó esta regla y la comunicó a Antonio María del Fiore, de Venecia, quien celebró un certamen con Niccolo Tartaglia, de Brescia, lo que dio ocasión a que Niccolo por sí mismo la descubriera, el cual me la dio a mí, suprimida la demostración, como consecuencia de mis ruegos. Pertrechado de este auxilio, busqué la demostración por varios caminos, lo que fue muy difícil.» 
Cardano se muestra desde luego sincero y agradecido con Tartaglia y los que le precedieron en la solución de la cúbica -ya en la introducción del Ars magna había hecho otro tanto-; excepto en un detalle: Cardano no dice que Tartaglia le dio, en verso, una indicación de como deducir la solución de la cúbica:

«Cuando está el cubo con las cosas preso

y se iguala a algún número discreto

busca otros dos que difieran en eso.

Después tú harás esto que te espeto que su producto siempre sea igual al tercio cubo de la cosa neto.

Después el resultado general de sus lados cúbicos bien restados te dará a ti la cosa principal»

([Martín Casalderrey, 2000: 117]).

El verso, aun no siendo cristalino, permitirá al lector, a poco que se lo proponga, encontrar la solución de la ecuación cúbica $x^{3}+p x=q$.

Al final del Ars Magna Cardano escribió: «Escrito en cinco años puede que dure varios siglos». Sin embargo, la obra envejeció pronto; por dos razones: por un lado, al carecer de una notación adecuada Cardano -al igual que todos sus contemporáneos - tuvo que usar un lenguaje retórico que quedó pronto anticuado al desarrollarse y consolidarse a principios del siglo XVII la notación que dio al álgebra toda la potencia de su generalidad -la diferencia se puede apreciar aquí, basta tener en cuenta que los dos primeros versos de Tartaglia «el cubo con las cosas preso se iguala a algún número discreto» eran la forma de la época de manejar la ecuación de tercer grado que hoy escribimos: $x^{3}+p x=q-$; por otro lado, la deficiente comprensión de los números negativos le hizo considerar una multiplicidad sin fin de casos - por ejemplo, para evitar los números negativos las ecuaciones $x^{3}+2 x-2=0$ y $x^{3}-2 x+2=0$, que hoy consideramos de la misma clase, eran consideradas en la forma $x^{3}+2 x=2$ y $x^{3}+2=2 x$ y discutidas como casos distintos-. En resumen, en el plazo de un siglo el Ars Magna se había convertido en un libro de lectura difícil y tediosa. Lo que no es óbice para considerar la resolución de las ecuaciones de tercer y cuarto grado como el gran descubrimiento matemático del Renacimiento -italiano, habría que añadir-. 
Cuatro efemérides matemáticas a la sombra de los griegos...

\section{Thăbit y Cardano}

A pesar de las diferencias de lugar y, sobre todo, de tiempo Thābit y Cardano presentan algunas similitudes que conviene resaltar. Digamos que estas similitudes afectan también a sus épocas respectivas. Podemos sin duda calificar de renacimiento al siglo IX en la Bagdad de Las mil y una noches en tanto en cuanto presenta algunos aspectos significativos comunes con el Renacimiento europeo del siglo XV, especialmente en lo referente a la recuperación de la cultura griega.

Remontándonos al Imperio Romano y haciendo una simplificación arriesgada podríamos decir que los bárbaros conquistadores de la zona occidental tomaron del Imperio su religión, esto es, el cristianismo, mientras que los que poco a poco fueron conquistando la zona oriental -los árabes en primera instancia- tomaron de este su cultura, esto es, la cultura clásica griega.

Durante el siglo IX, el siglo de Thābit, se produjo una recuperación de la ciencia y cultura griegas por parte de los árabes -que no afectó, a diferencia de lo que luego sería el Renacimiento italiano a las artes-; como se apuntó en la introducción, la vía fue esencialmente la traducción del griego al árabe de gran parte del legado griego; pero los árabes no sólo lo recuperaron sino que también lo absorbieron, lo hicieron propio y lo mejoraron. Por ejemplo, en lo relativo a la astronomía no sólo accedieron a las fuentes griegas, ya se habló antes de las traducciones del Almagesto a principios del siglo IX, sino también a los conocimientos hindúes en astronomía; crearon a su vez dos observatorios, primero en Damasco, en el siglo VIII, todavía la época omeya y, después, en Bagdad ya bajo el califa Al-Mamun (siglo IX).

Todo este proceso se repitió posteriormente en Europa Occidental de forma mucho más dilatada en el tiempo -las traducciones empezaron en el siglo XII y siguieron durante el XIII, el XIV y aún en el XV, sobre todo tras la caída de Constantinopla-, aunque acabó floreciendo durante el siglo XV con el Renacimiento italiano. Ya hemos dicho que en este caso el Renacimiento afectó también a la recuperación de la arquitectura y escultura, y todo ello devino también en un cambio de actitud ante la vida -terremoto vital que también, en forma de conversión al Islam, sacudió al mundo árabe en el siglo VII-.

Thābit y Cardano fueron fiel reflejo de sus épocas, y dado que sus épocas fueron en cierta forma similares, ellos lo fueron también: los dos fueron matemáticos, pero también médicos y filósofos y físicos, el uno fue también astrónomo mientras que el otro fue astrólogo. En fin, lo que se ha dado en llamar hombres del Renacimiento. Sin embargo, una reflexión 
sobre sus vidas nos convence también de que Thābit fue más científico que Cardano, en el sentido en que hoy entendemos el término científico. Donde más se nota esto que digo es en lo referente a los astros. Ya se ha dicho que Thābit fue astrónomo, lo que antes de mediados del siglo XVII casi también significaba astrólogo -piénsese en Ptolomeo, Brahe, Kepler, ...-. Curiosamente la obra de Thābit está libre de planteamientos astrológicos, si exceptuamos su libro sobre imágenes mágicas De imaginibus que es, por otro lado, más reflejo de su convicción de sabeo que de astrónomo -([Carmody, 1955: 242])-. Pero también es significativa su contribución a la matematización de la astronomía -sustituyendo algunos tratamientos empíricos presentes en Ptolomeo por modelos geométricos donde poder demostrar lo que antes habían sido meros asertos heurísticos- y, sobre todo, fue pionero en la exigencia de un cierto escepticismo para con la teoría a la espera siempre de nuevas confirmaciones procedentes de los datos de observación.

Nada de esto encontramos en Cardano. Desde luego su faceta de astrólogo va más allá de lo científicamente admisible, aun en su época. Aunque en esto, como en el resto, Cardano no fue más que fiel a su tiempo: no olvidemos que la recuperación del legado de los clásicos incluyó también la afición de estos por la astrología -sobre todo después de que Alejandro Magno la importara de oriente-. Y es que es imposible entender la personalidad -científica incluso- de Cardano sin entender cabalmente su época: el prodigioso Renacimiento excesivo tanto en creaciones como en miserias. No puedo evitar la tentación de usar la prosa evocadora del escritor ruso Dimitri Merezhkovski para ilustrar esto. Concretamente reproduciré unos párrafos de su novela El romance de Leonardo -da Vinci-. En ellos se narra una memorable escena: un banquete de Año Nuevo ofrecido por Ludovico Moro; Leonardo da Vinci ha colaborado creando varios ingenios mecánicos para divertimento de los comensales. En primer lugar se narran los prodigios de la época:

"Los invitados pasaron a otra sala en la que se exhibía un nuevo espectáculo. Tirados por negros, leopardos, grifos, centauros y dragones, los carros triunfales de Numa, Pompilio, César, Augusto y Trajano, se mostraban ornados de inscripciones alegóricas. Estos héroes eran los precursores de Moro. Por fin, apareció un carro, arrastrado por unicornios llevando un globo enorme que representaba la esfera celeste, sobre la que se reclinaba un guerrero con coraza de hierro. Un niño desnudo pintado de púrpura, llevando en la mano una rama de moral-moro en italiano-, salía por una abertura de la coraza del guerrero, lo que significaba el fin del siglo de hierro y el nacimiento, gracias al sabio gobierno de Moro, de un nuevo Siglo de Oro. Advirtieron, con general sorpresa, que la estatua de oro era un niño 
vivo. El pequeño, bajo la costra de oro que cubría su cuerpo, se encontraba molesto y las lágrimas brotaban de sus ojos».

Y ahora las sombras; a la mañana siguiente de la orgiástica fiesta:

«Leonardo se aproximó a la apagada chimenea [...]. De pronto apercibió en un rincón de la chimenea al niñito pintado de púrpura que dormía entumecido por el frío, hecho un ovillo, con la cabeza sobre las rodillas que rodeaba con sus brazos. Leonardo le tocó suavemente en un hombro. El niño, sin levantar la cabeza, gimió sordamente. El artista tomándole en brazos, lo apretó contra su pecho. El niño, con el rostro morado de frío, entreabriendo los ojos no pudo contener los sollozos. «iLlévame a casa, a casa!», «¿Dónde vives? ¿Cómo te llamas? -le preguntó Leonardo.» «Lippi-dijo el chiquillo-. ¡Oh! ¡Me duele el corazón! Tengo frío ... ¡Llévame a casa!» Sus párpados se cerraron $y$, delirando con voz cantarina, brotaron en sus labios las palabras del himno: «Volverá, gracias a Moro, el dichoso Siglo de Oro!». [...] El artista envolvió cuidadosamente a Lippi en su capa. Una vez fuera de palacio, dio un rodeo para entrar en una botica y comprar los ingredientes necesarios para quitar la dorada costra del cuerpo del chiquillo y llevarle a su casa. [...] El niño tiritaba de fiebre. «iNuestro Siglo de Oro», pensó el artista sonriendo tristemente. «Pobre pajarillo!»-murmuró observando al pequeño con infinita piedad. Y puso tanta emoción y ternura al apretarle contra su pecho, que el niño enfermo tuvo la sensación de que su madre muerta le acariciaba y mecía».

El niño acabó muerto, contaminado con la pintura y aterido de frío.

Así fue el Renacimiento y así fue Cardano: capaz de explicar cómo se resuelven las ecuaciones de tercer y cuarto grado, pero también de hacerse un horóscopo a sí mismo -por no hablar del de Jesucristo-, pronosticarse el día de su muerte para el 21 de septiembre de 1576 y jacertar! Morris Kline logró describir adecuadamente como las matemáticas de Cardano estuvieron contaminadas de Renacimiento: «las matemáticas no eran un método para él; eran un talento mágico especial y una forma de especulación cargada emocionalmente.»

\section{Pierre de Fermat}

La biografía de Fermat es tan insulsa como singular y ajetreada la de Cardano. Fermat nació el 20 de agosto de 1601 en Beaumont de Lomagne en una familia acomodada. Estudió leyes y acabó siendo jurista y parlamentario en Toulouse, puesto que ocupó hasta su muerte -pasando entonces el puesto a su hijo mayor-. Se casó a los treinta años y tuvo cinco hijos; llegó a escribir poesía en francés, latín y castellano. Nunca tuvo apuros económicos. Pierre de Fermat hizo matemáticas simplemente por afición. 


\section{Antonio J. Durán Guardeño}

Aunque inmediatamente debemos aclarar que eran unos tiempos aquellos -hasta el último cuarto del siglo XVII, más o menos- en los que señalar la diferencia entre ser aficionado a las matemáticas y ser profesional de ellas no es cuestión fácil. En realidad, es incluso difícil precisar lo que significaba el término matemáticas, pues coexistían diferentes formas, técnicas y modos de proceder claramente diferenciados entre sí y, a veces, difícilmente reconciliables. Sin ánimo de ser exhaustivo, se puede señalar a: (1) los algebristas retóricos -cosistas- al estilo de Cardano; (2) los que seguían el patrón geométrico griego -con Federico Commandino como principal representante- con renuncia implícita -explícita a menudo- a mezclarlo con el álgebra; (3) los que tomando como punto de partida la matemática griega pretendían introducir nuevos métodos que la potenciaran y, eventualmente, superaran -Vieta fue quizá el primero-; (4) los astrónomos. De hecho, la denominación matemático se aplicaba más a estos últimos que al resto, a los que comúnmente se llamaba geómetras. Los astrónomos eran los más profesionales - piénsese en Galileo nombrado por Cósimo II de Médicis primer filósofo y matemático de la Toscana a raíz de sus primeros descubrimientos con el telescopio, o en Brahe y Kepler los matemáticos imperiales de Rodolfo II-, mientras que entre los geómetras no eran infrecuentes los aficionados al estilo de Fermat -v.g. el inventor de los logaritmos: John Napier, un noble escocés-. Esta situación puede tener sus desventajas, pero tiene una ventaja que no conviene olvidar: al dedicarse por afición a las matemáticas eran más libres para elegir los problemas que querían estudiar y resolver, especialmente si, como era el caso de Fermat, se gozaba de una más que buena situación económica. Conviene no olvidar el impulso que dio Fermat a la teoría de números, la rama más pura de las matemáticas, frente a la falta de entusiasmo que mostraron la mayor parte, sino todos, sus contemporáneos -Pascal, Wallis, van Schooten, Huygens, ...-.

Digamos que en la clasificación anterior Fermat entraba de lleno en el grupo tercero. Por la influencia que el Arte analítico de Vieta ejerció en Fermat, podemos considerarlo discípulo de Vieta, aun cuando Fermat tuviera sólo dos años a la muerte de aquel. El Arte analítico señalaba que el estudio de los griegos $-\mathrm{y}$ volvemos nuevamente a encontrarnos con ellos- era el punto de partida para hacer matemáticas, aunque sólo el punto de partida.

Durante el siglo XVI acabó de publicarse todo el legado matemático griego que se había ido recuperando en siglos anteriores, entre ellos algunos autores que casi no fueron tratados por los árabes -Pappus y Diofanto, principalmente-. Así, y por señalar sólo los ejemplos más signifi- 
cativos, la edición príncipe de Arquímedes apareció en Basilea en 1544 -incluyendo los comentarios de Eutocio-. Los cuatro primeros libros de Las cónicas de Apolonio -los únicos conservados en versiones griegas-se imprimen por primera vez en 1537, pero hasta 1566 no aparece la importante versión de Commandino -hasta 1661 no se imprimieron los libros V al VII sólo conservados en versiones árabes y hasta 1710 no apareció la edición príncipe de Halley-. La primera impresión de los seis libros conservados de la Arithmética de Diofanto apareció en 1575, en edición y traducción -al latín-de Xylander. Finalmente, en 1589 apareció la primera impresión de la Colección Matemática de Pappus, en edición de Commandino. Esto supuso que los matemáticos de la generación de Fermat fueran los primeros de Occidente, no sólo en conocerlos, sino también, en dominarlos.

Hacía el final de la década 1620-1630, Fermat entró en contacto con el círculo de discípulos de Vieta en Burdeos -donde este había trabajado y donde, finalmente, murió-. Fermat aprendió de estos, y de los libros de Vieta, qué había que estudiar y, quizá no menos importante, cómo había que estudiar a los griegos. Especial influencia ejercieron en Vieta -y después en Fermat- la Arithmetica de Diofanto y, sobre todo, la Colección matemática de Pappus. Estos textos suponían una diferencia con la matemática griega anterior, en cuanto que daban información sobre cómo abordar la solución de problemas: aparecía en ellos un, digamos, análisis geométrico muy diferente a las deducciones sintéticas de teoremas al estilo de Los Elementos o las obras conocidas de Arquímedes. Esto fue también señalado por Descartes en la regla IV de las Reglas para la dirección del espíritu -escritas probablemente en el invierno de 1628-, aunque Descartes afirmó no haberlo tomado de Vieta a quien, aseguraba, no leyó hasta después de la publicación del Discurso del método (1637). Vieta había procedido también a una algebrización -Diofanto fue, en este caso, importante- de ese análisis geométrico griego buscando en la generalidad del álgebra un método más eficaz de investigación. El álgebra resultante -digamos mejor el arte analítico, pues Vieta consideraba bárbaro el término álgebra - fue más sofisticada que la italiana con ciertas mejoras en la notación -uso de vocales mayúsculas para las incógnitas y consonantes mayúsculas para los parámetros-; aunque arrastraba todavía la importante traba de la homogeneidad que delataba su origen geométrico griego, y la persistencia en el estudio de casos similares por el rechazo de los números negativos.

En manos de Fermat, las ideas seminales del Arte analítico de Vieta pronto germinaron en métodos más potentes. El primero, basado en la teoría de ecuaciones de Vieta, fue el de máximos y mínimos -que pudo te- 
ner más o menos listo hacia 1629, estando todavía en Burdeos-; de él derivaría después el método de tangentes -expuesto por primera vez en una carta a Mersenne en 1638-. En estas primeras versiones ambos métodos fueron aplicados a curvas algebraicas -digamos, en términos actuales, curvas definidas implícitamente por una ecuación polinómica en dos variables-. Algo más tarde, hacía 1636, Fermat conseguiría con su geometría analítica lo que Vieta quiso pero no pudo lograr.

Conviene aquí hacer una pausa en la producción matemática de Fermat para comentar uno de los rasgos más significativos de su carácter de aficionado: la no publicación de sus trabajos. Porque Fermat nunca llegó a publicar, en vida, ninguno de sus resultados: fue su hijo mayor quien publicó póstumamente -1674- gran parte de la obra matemática de Fermat -antes, en 1670, había hecho una edición del ejemplar anotado por Fermat de la Arithmética de Diofanto, editada por Bachet de Méziriac, que incluía la formulación de su célebre teorema/conjetura-. En cualquier caso, buena parte de sus trabajos se habían difundido prácticamente por toda Europa a través del círculo de Mersenne, con quién Fermat entró en contacto hacia 1636 por mediación de Pierre de Carcavi -la primera carta conservada lleva fecha de 26 de abril de 1636-. Por más que Fermat fuera urgido a publicar sus trabajos, nunca lo hizo. Y no es que Fermat quisiera pasar desapercibido -la comunicación con Mersenne y sus amigos garantizaba a sus trabajos una aceptable difusión-, más bien era muy remiso a realizar el trabajo de revisión, ordenación y pulido necesario para la publicación -digamos que esta faceta aburría a Fermat que prefería dedicar su esfuerzo a la más excitante tarea de buscar la solución de los problemas-. Esto tuvo como contrapartida la pérdida de influencia de Fermat en los matemáticos de la segunda mitad del siglo XVII y, naturalmente, en los que siguieron -con la única excepción de la teoría de números, como después se verá-; también le supuso cierta pérdida sobre la paternidad de sus resultados. Es desde luego el caso de la geometría analítica, normalmente atribuida a Descartes, aunque Fermat enviara su versión -que tituló Ad locos planos et solidos isagoge- a Mersenne a finales de 1636 o principios de 1637, antes, pues, de la publicación del Discurso del método cartesiano y sus célebres apéndices. En este caso, el triunfo de Descartes también se explica por la mejor notación que desarrolló frente al excesivo apego que mantuvo Fermat a la de Vieta, lo que hizo su versión -y en general sus otros trabajos- más difíciles de seguir y entender. La aparición de ambas geometrías provocó un enfrentamiento epistolar entre Fermat y Descartes. No fue exactamente por motivos de prioridad en el descubrimiento, aunque algo hubo de eso. Se inició por unas críticas de Fermat a la Dióptica cartesiana; la res- 
puesta de Descartes fue áspera -celoso quizá por la pérdida de protagonismo que la aparición de la geometría analítica de Fermat ocasionaría a la suya- y su crítica afectó a prácticamente todos los trabajos conocidos de Fermat: sus matemáticas, arguyó Descartes, no tenían método, ni generalidad, ni formaban un sistema; eran, en definitiva, poco más que suerte. La perdedora en la disputa fue la fama de buen matemático que por entonces ya se había ganado Fermat que se vio algo dañada; para recuperarla Fermat revisó sus métodos de máximos y mínimos y tangentes haciéndolos más eficaces con aplicaciones exitosas a curvas transcendentes -cisoide, cicloide, conchoide, cuadratriz-. Aunque estos métodos de Fermat no estaban basados en los infinitesimales -no son necesarios para curvas algebraicas y, para las trascendentes, Fermat los evitó sustituyendo longitudes de arco por la parte correspondiente de las tangentes- son un antecedente del cálculo diferencial, en el que Newton reconoció haberse inspirado para desarrollar su método de fluxiones. Aunque todo esto no es suficiente para nombrarlo, como hicieron algunos matemáticos franceses del XVIII, inventor del cálculo.

Y seguiría sin ser suficiente si añadimos las contribuciones de Fermat al cálculo de áreas y volúmenes, centros de gravedad o rectificación de curvas. De nuevo aquí tomó Fermat como punto de partida la obra de un griego: el maestro Arquímedes, en este caso. Y, también, acabó desarrollando métodos más potentes basados en la aritmética, el álgebra y los procesos infinitos.

Los procesos infinitos aparecen por primera vez en la obra de Fermat en relación con las cuadraturas; ya comenté que no los hay en los máximos y mínimos y tangentes, aunque bien pudieran haber aparecido si Fermat los hubiera vuelto a revisar -no volvió a ellos después de 1644una vez atravesó la barrera de lo infinitesimal en sus trabajos sobre áreas. Junto con la geometría analítica, los infinitesimales -el infinito en acto- fueron la gran aportación matemática del siglo XVII que permitieron a los matemáticos superar definitivamente el legado griego. La superación en el siglo XVII del horror infiniti que pareció aquejar al mundo griego tuvo que ver, y mucho, con las consideraciones teológicas sobre el infinito en acto - prohibido por Aristóteles-. Estas fueron tratadas minuciosamente por los escolásticos -recuérdese lo comentado en relación con Thābit y el infinito- y aparecen con bastante frecuencia en los filósofos del siglo XVII -no se olvide que algunos de aquellos filósofos fueron también matemáticos-. Así lo encontramos en Descartes: «Por Dios entiendo una sustancia infinita, eterna, inmutable, independiente, omnisciente, omnipotente», y también: «Reservamos solamente para Dios la calificación de infinito, tanto en razón de que no observamos límites en sus 
perfecciones, como también a causa de que estamos muy seguros de que no puede tenerlos»; en Spinoza: «Entiendo por Dios un ser absolutamente infinito, es decir, una sustancia constituida por una infinidad de atributos, cada uno de los cuales expresa su esencia eterna e infinita»; y también en Leibniz: «Cabe juzgar que esa Sustancia Suprema, que es única, universal y necesaria debe ser incapaz de límites y contener tanta realidad cuanta sea posible». ${ }^{5}$

De mano de los procesos infinitesimales y la generalidad del álgebra -aplicada a la geometría mediante la geometría analítica-, la matemática durante el siglo XVII acabó derivando de una resolución de problemas específicos a métodos de solución, culminando con la síntesis del cálculo infinitesimal por Newton y Leibniz. Y, desde luego, Fermat es una figura clave en esta transición de las matemáticas griegas a las modernas. Fermat entendió que las matemáticas debían ser mucho más que una admiración por los esplendores pasados -la matemática griega en su caso-, debían consistir en un avance continuo buscando la resolución de problemas -él siempre ha tenido fama de ser lo que en inglés se llama un problem solver-para lo que era imprescindible el desarrollo de técnicas nuevas que garantizaran el progreso perpetuo de este arte. En este sentido hay que añadir que Fermat estuvo presente, en mayor o menor medida, en todas y cada una de las grandes teorías matemáticas -salvo la geometría proyectiva- que surgieron, o resurgieron, durante el prodigioso siglo XVII: la geometría analítica, el cálculo infinitesimal, el cálculo de probabilidades y la teoría de números.

La afición de Fermat por la teoría de números tiene de nuevo un griego detrás, en este caso Diofanto y la edición de Bachet de Méziriac de su Arithmética -la primera incluyendo el texto en griego-: precisamente a un ejemplar de esta edición corresponde el margen demasiado estrecho para contener la demostración del gran teorema / conjetura que ha dado a Fermat más fama que todos sus demás logros juntos.

En cierta forma, Fermat fue el creador de la teoría de números como rama independiente de las matemáticas, y fue, de todas las áreas en las que trabajó, donde su influencia se notó más; aunque esta influencia no se debe a sus contribuciones sino más bien al estímulo que misteriosamente supo ejercer sobre las generaciones que le siguieron. Y digo misteriosamente porque a ningún matemático contemporáneo de valía logró contagiar Fermat su amor por la teoría de números, y no fue por falta de intentos: ahí están sus cartas a Pascal, Wallis, Van Schooten y Huygens -estos últimos consideraron la teoría de números carente de interés-. Esto a la postre fue una ventaja para Fermat porque nadie acabó publicando un texto que anulara la influencia de los resultados que él mal con- 
taba en cartas o márgenes -como le ocurrió con el resto de su producción-. Más bien al contrario, si atendemos al éxito de interés acumulado por su célebre teorema/conjetura: su solución hace algunos años ha sido de los escasísimos logros matemáticos que han logrado llegar a los media.

Más que detallar la producción de Fermat en teoría de números intentaré responder a la pregunta: ¿Era Fermat sincero cuando decía que tenía la demostración de un resultado que establecía como teorema? A pesar de las apariencias -y de las frustraciones que generó entre matemáticos e historiadores posteriores- yo me inclinaría a decir que sí, que cuando decía tener una demostración la tenía o, al menos, creía que la tenía. Alguien podría decirme que se ha demostrado la falsedad de algunos resultados conjeturados por Fermat y por lo tanto no debía de tener ninguna demostración de ellos; por ejemplo, Fermat conjeturó que los números de la forma $2^{2^{n}}+1$ son primos y Euler probó en 1732 que para $n=5$ se obtiene el número 4.294.967.297 que admite la factorización 641x6.700.417. Este ejemplo no vale porque Fermat nunca aseguró tener una demostración: "Estoy casi persuadido», escribió a Bernard de Frenicle, «he excluido un número tan grande de divisores por un método infalible que me sería difícil retractarme». Todos los teoremas de Fermat, aquellos de los que dijo disponer de una demostración, han sido finalmente demostrados: incluido su gran teorema, aunque en este caso se hayan necesitado más de tres siglos y medio y el esfuerzo de muchas generaciones de matemáticos para lograrlo. ¿Es razonable pensar, entonces, que Fermat disponía de una demostración maravillosa, aunque el margen de la Arithmética de Diofanto era insuficiente para contenerla? Digamos que es razonable pensar que Fermat lo creía así. La conjetura de Fermat establece que la ecuación $x^{n}+y^{n}=z^{n}$ no admite soluciones enteras para $x, y, z$, cuando $n$ es mayor que 2 . Pues bien, usando su método de descenso infinito Fermat pudo haber demostrado su conjetura para los valores $n=3$ y 4 : la demostración del caso $n=4$ está, de hecho, casi implícita en otro margen del libro donde mostró por descenso infinito que la ecuación $x^{4}+y^{4}=z^{2}$ no tiene soluciones enteras; y del caso $n=3$ ya nos enseñó Euler como pudo ser la demostración de Fermat usando descenso infinito. A partir de estos casos -que Fermat cita numerosas veces en su correspondencia mientras que el caso general sólo aparece en el célebre margen- pudo caer en la tentación de pensar que había encontrado el principio general para una demostración; y es que, si atendemos a la afortunada frase de André Weil: ¿cómo podía adivinar Fermat mientras escribía en aquel estrecho margen de la Arithmetica de Diofanto que lo estaba haciendo para la eternidad? 
Tres generaciones de matemáticos pasaron antes de que la teoría de números fuera otra vez considerada seriamente: en Euler se reencarnó, finalmente, el amor de Fermat por la teoría de números. Luego vinieron, Lagrange, Legendre y, sobre todo, Gauss y su portentoso cerebro.

\section{Las Disquisitones Arithmeticae de Gauss}

Todavía se conserva el prodigioso objeto que alcanzó esa excepcional cumbre matemática de la que este año celebramos el doscientos aniversario de su publicación. El prodigioso objeto es el cerebro de Gauss y la cumbre matemática las Disquisitiones arithmeticae. El cerebro de Gauss es el principal adorno -permítaseme decirlo así- de la colección anatómica de la Universidad de Gotinga; allí se guarda la inquietante colección de sesos que Rudolf Wagner, un craneometra que vivió durante el siglo XIX, reunió de entre el profesorado de dicha Universidad. Wagner, en la línea de Paul Broca, pretendía demostrar la relación directa entre el tamaño del cerebro de un individuo y su grado de inteligencia -una versión intelectual, podríamos decir, del más popular debate sexual sobre la importancia del tamaño del pene-. En cualquier caso, el cerebro de Gauss no es, en cuestión de tamaño, demasiado sorprendente -1.492 gramos, poco más grande que la media-, aunque sí es muy intrincado y retorcido -cuando digo retorcido sólo quiero decir excepcionalmente pródigo en circunvoluciones-. El tamaño del cerebro de Gauss supuso una cierta desilusión para Wagner, Broca y sus seguidores -aunque no fue la peor-. Sin embargo, el cerebro conservado no fue exactamente el objeto que engendró las Disquisitiones: el conservado es el cerebro del anciano Gauss muerto a los 78 años, mientras que su obra cumbre en teoría de números fue gestada y alumbrada por el cerebro de un joven Gauss con poco más de 20 años. Todos sabemos que las neuronas no se dividen y que, por tanto, las que le quedaran a Gauss al final de sus días ya estaban presentes en su cerebro de 20 años, pero hubo otras que no resistieron el paso del tiempo marcando con su muerte diferencias cerebrales entre el Gauss de 78 y el de 20 años; pero, sobre todo, esas diferencias las acrecentaron los diferentes senderos y caminos que las vicisitudes de su vida fueron trazando entre las redes neuronales de su cerebro; ese que, apropiándose del título de un cuento de Borges, se puede calificar de jardin de senderos que se bifurcan.

No querría proyectar una imagen de Gauss demasiado romántica dado que él, a pesar de vivir en pleno romanticismo, no lo fue; como tampoco fue revolucionario en un época llena de revoluciones. En este senti- 
do no estuvo tan marcado como Cardano por su tiempo -habría que decir mejor, por lo nuevo de su época-. Gauss tuvo, en cierta forma, una mentalidad más dieciochesca. Nunca le atrajeron las revoluciones -más bien le espantaron-. Se acomodó bien al sistema feudal político en el que vivió que, por otro lado, no lo trató mal: el duque de Brunswick le pagó sus estudios, primero en Brunswick y, luego, en Gotinga -a pesar de que Gauss fue allí contra los deseos del duque-, y lo mantuvo, después, hasta que fue nombrado Director del Observatorio de Gotinga-Gauss le dedicaría al duque las Disquisitiones en una cargada dedicatoria que comienza: "Considero mi mayor fortuna que V.E. me permitiera adornar este mi trabajo con su honorabilísimo nombre» [Gauss, 1981: 3]-. Esa estructura del mundo en el que Gauss se sentía bien se vino abajo como consecuencia de las invasiones napoleónicas, lo que siempre le generó fobia por cualquier tipo de revolución y, de paso, por los franceses.

Pero vayamos con el objeto de la efemérides: las Disquisitiones arithmeticae, todo un clásico de la ciencia que, como suele ocurrir con los clásicos, son muchos los que lo citan pero pocos los que lo han leído -y menos aún los que lo han estudiado, aunque se trata, en este caso, de un clásico de lectura seca y difícil-. Aparecieron en septiembre del primer año del siglo XIX; Gauss publicó el libro en latín -un latín refinado que le revisó $\mathrm{F}$. Meyerhoff, un amigo de la infancia-, como la mayor parte de sus trabajos posteriores, a pesar de que era una costumbre ya algo desfasada por esa época - un indicativo más de la mentalidad dieciochesca de Gauss-. Por cierto que Gauss tuvo en mente publicar una segunda parte de las Disquisitiones -así consta en algunos fragmentos de su correspondencia- lo que nunca se produjo; en parte porque Gauss ya jamás le dedicaría tanto tiempo y energías a la teoría de números como en los años que estudió en Gotinga (1795-1798), donde se gestó gran parte de la obra -aunque sí publicó con posterioridad algunos artículos sueltos sobre el tema-.

Las Disquisitiones iniciaron, con el nuevo siglo, un nuevo periodo de esperanzador futuro para la teoría de números que pasaba a contar con un estudio sistemático que englobaba y, sobre todo, extendía gran parte de los resultados brillantes, pero aislados, logrados por un puñado de matemáticos durante el siglo y medio anterior: primero Fermat que, según comentamos más arriba, impulsó definitivamente y en solitario la teoría hasta que, un siglo después, se unieron Euler, Lagrange y Legendre -junto con Diofanto, son los ilustres nombrados por Gauss en el prefacio de las Disquisitiones-.

A Gauss, la publicación le supuso también alcanzar una gloria matemática difícilmente esperable a su corta edad -hasta ese momento tan 
sólo había publicado un adelanto de la construcción del polígono regular de 17 lados y su tesis: la primera demostración, razonablemente aceptable, del teorema fundamental del álgebra-; aunque a Gauss esta gloria no le sorprendió: seguramente por las veredas de ese su joven cerebro de 20 años ya llevaba circulando algún tiempo la sospecha de que al igual que Arquímedes y Newton, él sería también un illustrissimus de las matemáticas.

El mismo mes de la publicación de las Disquisitiones logró otro éxito que llevaría su fama más allá del universo de los matemáticos: la localización del asteroide Ceres perdido tras haber sido observado por primera vez el 1 de enero de 1801 por el astrónomo Giuseppe Piazzi. Las Disquisitiones y los cálculos astronómicos que le permitieron localizar a Ceres conforman un ejemplo magnífico para ilustrar la manera en que Gauss entendió las matemáticas: eran una ciencia - la reina de las ciencias, según sus propias palabras- con dos caras: por un lado era un juego del espíritu, por otro una herramienta básica para el estudio y comprensión de la naturaleza.

Gauss tenía ya hacia 1797 un borrador casi definitivo de las cuatro primeras secciones de las Disquisitiones -él y su cerebro tenían entonces 20 años-. Las tres primeras secciones son de carácter introductorio: conceptos y resultados elementales sobre congruencias y residuos, incluyendo la factorización en primos de los números enteros -implícita ya en Los Elementos de Euclides-, el teorema pequeño de Fermat y el de Wilson, por poner algunos ejemplos. La cuarta sección incluye la primera demostración de la ley de reciprocidad cuadrática -según Gauss esta ley es «una de las más elegantes de su género» ([Gauss, 1981: 117])-. Como el mismo Gauss explica ([Gauss, 1981: §151]), la Ley se remonta a Euler que fue el primero en apuntarla, pero sin prueba; Legendre había propuesto una primera demostración en 1785, pero como él mismo confesó, basada en resultados no demostrados -Gauss incluyó otra demostración de la Ley en la sección V-.

La sección $\mathrm{V}$ es la más voluminosa de todo el libro -ocupa las tres quintas partes-. Está dedicada a la teoría de formas cuadráticas binarias que comenzara a estudiar Lagrange tres décadas antes y, en menor medida, cuadráticas ternarias -grosso modo estudia cómo representar números mediante expresiones de la forma $a x^{2}+2 b x y+c y^{2}$ (binarias) y $a x^{2}+2 b x y+2 c x z+2 d y z+e y^{2}+f z^{2}$ (ternarias)-; y esto sin contar la sección VI que es, en cierta manera, un apéndice de la V. Una parte de la sección V desarrolla la manipulación algebraica de estas formas -incluyendo las definiciones de orden, género y carácter, y la composición de formas; todo con gran influencia posterior-. Sin embargo, lo que le interesaba a Gauss 
no era el álgebra de las formas sino su aplicación a la resolución de problemas de la aritmética; estas aplicaciones completan la sección: se solucionan diversas ecuaciones diofánticas, se da otra demostración del teorema de reciprocidad cuadrática, se estudia la representación de números como suma de tres cuadrados, se demuestra que todo número admite una descomposición como suma de 3 números triangulares ${ }^{6} \mathrm{y}$, también, como suma de a lo más cuatro cuadrados -resultados que había enunciado Fermat y todavía estaban pendientes de demostración-, etc. Digamos que un primer borrador muy parcial de estas secciones pudo estar listo hacia el verano de 1796, en el invierno de 1798-1799 se añadió lo referente a las formas ternarias y hacia 1800 pudieron estar definitivamente listas. Hay datación precisa para algunos de los resultados; la descomposición en suma de tres números triangulares aparece anotada en el diario de Gauss en la forma: «EYREKA! Num $=\Delta+\Delta+\Delta »$, y lleva fecha 18 de julio de 1796 -sí, exactamente ciento cuarenta años antes de la sublevación de Franco-.

La sección VII y última es bastante homogénea y autocontenida con respecto al resto - de hecho fue escrita antes de que hubiera una versión definitiva de la $\mathrm{V}-$, y podría decirse que es la más popular, si no fuera atrevimiento usar este calificativo para otro resultado matemático que no sea el teorema de Pitágoras. Está dedicada a las ecuaciones ciclotómicas y la descomposición con regla y compás de la circunferencia en partes iguales -esto es, la construcción con regla y compás de polígonos regulares-. Las construcciones con regla y compás pueden escribirse en términos algebraicos: aquellas requieren intersecciones de rectas, rectas y circunferencias, o dos circunferencias, lo que equivale algebraicamente a la resolución de sistemas de ecuaciones lineales, lineal y cuadrático o, dos ecuaciones cuadráticas.

El resultado estrella de la sección VII demuestra que siempre se puede construir, con regla y compás, un polígono regular de $n$ lados cuando $n=2^{n} p_{1} p_{2} \ldots p_{k}$, donde $p_{1}, p_{2}, \ldots, p_{k}$ son números primos de Fermat -esto es, números primos de la forma $2^{2^{n}}+1$. Gauss apunta también aunque sin demostrarlo - «los límites de este trabajo excluyen esta demostración aquí", escribe ([Gauss, 1981: 462])- que esa forma particular del número $n$ no es sólo suficiente sino también necesaria.

$\mathrm{Y}$, como aplicación, Gauss muestra la forma en que puede ser construido un polígono de 17 lados $-2^{2^{2}}+1$ lados-. Como se dijo en la introducción, Gauss se remontó a los griegos para darle solera y prestancia a una construcción que, según reza su diario, descubrió el 30 de marzo de 1796. Fue, por tanto, el fruto asombroso de un prodigioso cerebro de tan sólo 19 años. 


\section{Antonio J. Durán Guardeño}

\section{Notas}

1 Dos números se dicen amigos si cada uno es suma de los divisores propios del otro -divisores propios de un número: los divisores excluido el número-. Por ejemplo el 220 y el 284 -son los dos números amigos más pequeños-; en efecto, $284=2^{2} \times 71$ y $220=2^{2} \times 5 \times 11$, de donde se obtiene que los números $1,2,4,71$ y 142 son los divisores de 284, mientras que $1,2,4,5,10,11,20,22,44,55$ y 110 son los de 220 . La amistad de 220 y 284 es ahora fácilmente deducible: $220=1+2+4+71+142$ y $284=1+2+4+5+10+11+20$ $+22+44+55+110$.

2 Un número perfecto es aquel que es suma de sus divisores propios. Por ejemplo: el $6=1+2+3$.

3 Aún hoy (2001) son muchas las cosas que no sabemos de los números amigos: por ejemplo, si hay o no una cantidad infinita de ellos.

${ }_{4}$ Sí suponen sus manipulaciones un antecedente claro del ejemplo que traería a colación Galileo en sus Discorsi -cambiando pares por cuadrados-, aunque el sabio toscano extrajera conclusiones harto diferentes a las de Thābit.

5 Conviene aquí, aunque sólo sea por reducirle el ego a Dios y aliviarse uno de tanta loa a su poderosa esencia, citar la minimalista definición de María Zambrano: «la nada es Dios".

${ }^{6}$ Los números triangulares tienen la forma $n(n+1) / 2$. El nombre deriva de que precisamente con $n(n+1) / 2$ puntos se puede formar un triángulo de puntos teniendo en la base justamente $n$ puntos -igual que con $n^{2}$ puntos se puede formar un cuadrado de puntos teniendo en la base justamente $n$ puntos-. La concepción geométrica de los números deviene naturalmente de la filosofía pitagórica.

\section{Bibliografía}

1 Bachmann, P.: Ueber Gauss' zahlentheoretische arbeiten, en C.F. Gauss Werke X,2, Georg Olms Verlag, Hildesheim. 1981.

2 BERry, A.: A short history of astronomy, Dover, New York. 1961.

3 Boyer, C. B.: Fermat and Descartes, Scripta Math., 18 (1952), 189-217.

4 Boyer, C. B.: Clairaut le cadet and a theorem of Thābit ibn Qurra, Isis, 55 (1964), 6870.

5 BÜHLeR, W. K.: Gauss, a biographical study, Springer Verlag, Berlín, 1981.

6 Cardano, G.: Mi vida, Alianza, Madrid. 1991.

7 CARmody, F. J.: Notes on the astronomical works of Thābit ibn Qurra, Isis, 46 (1955), 235-242.

8 Cid, F.: Historia de la ciencia, Planeta, Barcelona, 1977.

${ }^{9}$ Crombie, A. C.: Historia de la ciencia: de San Agustín a Galileo, Alianza Editorial, Madrid. 1993.

10 DreYer, J. L. E.: A history of astronomy from Thales to Kepler. Dover, New York. 1953.

11 Gauss, C. F.: Disquisitiones arithmeticae, en C.F. Gauss Werke I, Georg Olms Verlag, Hildesheim. 1981.

12 Gillispie, CH. C. (Editor): Dictionary of Scientific Biography, Scribner's \& Sons. Nueva York, 1981. Voces: Thäbit, Cardano, Fermat y Gauss.

13 Heat, T. L.: A History of Greek Mathematics. 2 Vols. Dover. New York, 1981. 


\section{Cuatro efemérides matemáticas a la sombra de los griegos...}

14 HogendiJK, J. P.: Thābit ibn Qurra and the pair of amicable numbers 17296, 18416, Historia Math., 12 (1985), 269-273.

15 Jensen, C.: Pierre Fermat's method of determining tangents of curves and its application to the conchoid and quadratrix, Centaurus, 14 (1969), 72-85.

16 Kline, M.: El pensamiento matemático de la Antigüedad a nuestros días. Vol.1. Alianza Universidad. Madrid, 1992.

17 Mahoney, M. S.: The mathematical career of Pierre de Fermat, Princeton University Press, Princeton. 1994.

18 Mahoney, M. S.: Fermat's mathematics: proofs and conjectures, Sciences, 178 (1972), 30-36.

19 Martin Casalderrey, F.: Cardano y Tartaglia: las matemáticas en el Renacimiento italiano, Nivola, Madrid. 2000.

20 MerezkHovski, D.: El romance de Leonardo, Círculo de Lectores, Barcelona. 1998.

21 MoRELON, R.: Thābit b. Qurra and arab astronomy in the 9th century, Arabic Sciences and Philosophy, 4 (1994), 111-139.

22 Rioja, A. y ORDóñez, J.: Teorías del universo. Vol. I: De los pitagóricos a Galileo. Vol. II: De Galileo a Newton. Síntesis, Madrid. 1999.

23 SAYILI, A.: Thābit ibn Qurra's generalization of the Pythagorean theorem, Isis, 51 (1960), 35-37.

24 ThĀBIT IBN QURRA: Oeuvres d'astronomie, Ed. trad. y comentadas por R. Morelon, Sciences et Philosphie arabes, Textes et études, París, 1987.

25 WEIL, A.: Number theory, an approach through history from Hammurabi to Legendre, Birkhäuser, Boston, 2001. 